\title{
Rechallenge of carboplatin-gemcitabine based chemotherapy for rapidly progressing metastatic collecting duct carcinoma of the kidney leading to a delayed and durable complete response: A case report
}

\author{
DOMITILLE DANO ${ }^{1}$, CLÉMENCE DELTEIL $^{2}$, ROMAIN BOISSIER $^{3}$, VÉRONIQUE DELAPORTE $^{3}$, \\ PAUL HABERT $^{4}$, SÉBASTIEN SALAS ${ }^{1}$, FLORENCE DUFFAUD ${ }^{1}$ and JEAN-LAURENT DEVILLE ${ }^{1}$ \\ Departments of ${ }^{1}$ Medical Oncology, and ${ }^{2}$ Pathology and Neuropathology, Assistance Publique-Hôpitaux de Marseille, \\ Timone University Hospital; Departments of ${ }^{3}$ Urology and Renal Transplantation and ${ }^{4}$ Radiology, \\ Assistance Publique-Hôpitaux de Marseille, Conception University Hospital, 13385 Marseille Cedex 5, France
}

Received July 25, 2018; Accepted November 29, 2018

DOI: $10.3892 / 01.2019 .9991$

\begin{abstract}
Renal collecting duct carcinoma (CDC) is a rare and highly aggressive subtype of kidney cancer with poor prognosis. We report a case of one patient, who was successfully treated with gemcitabine-platin based chemotherapy for polymetastatic renal CDC, and experienced a late and prolonged complete remission. In June 2014, a 69-year-old male patient was diagnosed with non-metastatic renal CDC. Nephrouretectomy was firstly performed. In December 2014, he developed a loco-regional recurrence with bilateral lung metastases. The patient started a course of gemcitabine-carboplatin (GC)-based first-line chemotherapy and received 6 cycles, which ended in May 2015. Computed tomography (CT) scan evaluation displayed an objective response according to RECIST 1.1 criteria and a follow-up of the patient was conducted. In August 2015, he had a second local relapse with new lung metastases. Despite a short disease-free interval, 6 cycles of the same GC regimen were required, which ended in February 2016. The patient firstly exhibited a partial objective response after the first 3 cycles and a stable disease at the end of chemotherapy. During the follow-up, a CT scan of his chest, abdomen and pelvis was performed every 3 months. From September 2016 to May 2017, despite no new specific treatments for his metastatic disease, the patient again experienced an objective and confirmed response on each CT-scan evaluation until complete remission in May 2017. This case report highlights the efficacy of GC-based chemotherapy,
\end{abstract}

Correspondence to: Dr Jean-Laurent Deville, Department of Medical Oncology, Assistance Publique-Hôpitaux de Marseille, Timone University Hospital, 264 Rue Saint Pierre, 13385 Marseille Cedex 5, France

E-mail: jean-laurent.deville@ap-hm.fr

Key words: renal collecting duct carcinoma, bellini carcinoma, complete remission, chemotherapy which is able to provide a durable and sometimes complete response in metastatic renal $\mathrm{CDC}$, and suggests the potential of rechallenging with the same chemotherapy regimen, despite a short disease-free interval. The originality of this case was demonstrated by the delayed complete response more than one year after the end of GC-based second line chemotherapy. The patient remained disease-free at his last CT-scan evaluation in April 2018.

\section{Introduction}

Renal collecting duct carcinoma (CDC), also known as Bellini carcinoma, is a rare and highly aggressive subtype of kidney cancer. Accounting for less than $1 \%$ of all renal cell carcinomas (RCC), patients with CDC have a poor prognosis (1).

At diagnosis, at least $50 \%$ of patients have synchronous node, bone or lung metastases (2). Median overall survival in metastatic patients is 6 months and systemic chemotherapy is only palliative $(3,4)$.

A total of $98.8 \%$ of non-metastatic patients undergo radical nephrectomy which can be curative in this setting (5). Currently, adjuvant treatment with radiation therapy and/or chemotherapy is not recommended. After surgery, clinical and imaging follow-up are required.

In metastatic renal CDC, there is no well-established standard-of-care. If nephrectomy remains standard in some metastatic clear-cell renal carcinomas, it seems useless in metastatic renal CDC, except for palliative indications (for example, pain, and uncontrolled hematuria) (3).

Moreover, a low level of evidence supports current systemic therapies performed in advanced renal CDC.

Until 2007, in comparison with urothelial carcinomas which share some pathological characteristics with CDC, MVAC chemotherapy (methotrexate, vinblastine, doxorubicin and cisplatin) was performed.

More recently, published data suggested that metastatic CDC might respond to gemcitabine and platin salt (GC) combination (4-6). 
Oudard et al reported results of a phase II clinical trial assessing gemcitabine/platin-based chemotherapy in first-line treatment of 23 patients with metastatic renal CDC. The objective response rate was $26 \%$ with 1 complete response (CR) and 5 partial responses. Median progression free survival (PFS) and median overall survival (OS) were respectively 7.1 and 10.5 months (6).

Currently, as underlined by Dason et al, only palliative GC based-chemotherapy could be recommended (7).

Two studies (one phase II single arm trial with 5 patients and one case report) suggested that addition of bevacizumab to platinum-based chemotherapy could improve efficacy of chemotherapy. These preliminary results need to be confirmed $(8,9)$. Bevacizumab is still under evaluation in combination with GC based-chemotherapy in metastatic renal CDC [BEVABEL GETUG-AFU phase 2 Trial (NCT02363751)].

One case report suggested that cabozantinib could be an active drug in metastatic renal CDC (10). A prospective phase 2 clinical trial with cabozantinib (NCT03354884) is currently recruiting patients.

Similarly to urothelial cancer, overexpression/amplification of human epidermal receptor-2 (HER2) was reported in CDC (11). The presence of this target may lead to alternative treatment options, such as trastuzumab but prospective data are needed before considering this approach.

Even if the results of HER2-targeted therapies in metastatic urothelial carcinoma were disappointing, Bronchud et al published a clinical and radiological response with a double-HER2 blockade in a patient with advanced CDC showing HER-2 overexpression (12).

Another retrospective work suggested that targeted therapies could play a role in selected cases of metastatic CDC of the kidney (13).

Here, we report the case of one patient successfully treated with gemcitabine-platin based chemotherapy for polymetastatic renal $\mathrm{CDC}$, and who experienced a late and prolonged complete remission.

\section{Case report}

A 69-year-old man with a history of diabetes, essential hypertension, coronary artery disease and peripheral vascular disease, was admitted to our center (AP-HM La Conception and La Timone University Hospitals, Marseille, France) in March 2014 to investigate an 8 centimeters renal tumor, localized in the left kidney hilum. The patient was asymptomatic and renal tumor was fortuitly diagnosed.

CT scan concluded to a left kidney tumor measuring $8 \mathrm{~cm}$, heterogeneously enhanced after iodine contrast, extending to the renal sinus and left psoas muscle (Fig. 1).

CT scan of chest-abdomen and pelvis did not display any evidence of metastatic disease and a nephroureterectomy was indicated after collegial discussion.

The surgical procedure was performed on June 2014 without any perioperative complications. Pathological analysis revealed a white, firm tumor measuring $10 \mathrm{~cm}$ long axis with necrosis, localized in the medulla with an involvement of renal cortex. One satellite nodule was observed in the perirenal fat. Histopathological findings showed a tubular proliferation associated with a desmoplastic response in the adjacent
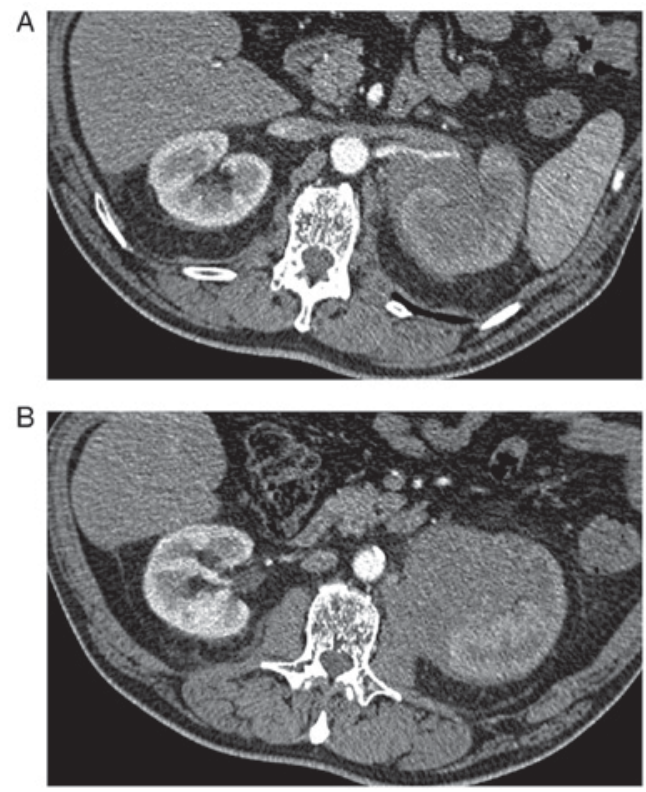

Figure 1. CT scan at diagnosis in March 2014. Axial contrast-enhanced CT scan show a left renal mass, which is infiltrative, centered on the renal medulla (A) eroding the left renal artery and with a delayed nephrogenic enhancement (B). At the lower part of the lesion there is a local invasion of the psoas muscle. CT, computed tomography.

stroma (Fig. 2A). There were irregular and branching tubules lined by a single layer of epithelium. The cells were cuboidal or hobnail cells with prominent nucleoli (cytologically high-grade) (Fig. 2B). The cytoplasm was clear. Mitosis were numerous and abnormal. Ancillary immunohistochemistry (Fig. 2C-F) showed an intense positivity of Cytokeratin 19 and Cytokeratin 7, a nuclear positivity of PAX8, a negativity of P63 and an absence of expression loss of INI-1 (1Nl-1 conserved). After eliminating a digestive origin, another renal cell carcinoma subtypes and urothelial carcinoma, pathologists concluded to a collecting duct carcinoma, with pT3 pN0 M0 stage. This diagnosis was confirmed after central pathology review in the INCA (National Cancer Institute of France)-labeled CARARE network.

The patient was then followed alternatively by ${ }^{18} \mathrm{FDG}$ PET/CT in August 2014 and CT scan of chest, abdomen and pelvis in December 2014. In December 2014, 6 months after surgery, he developed a loco-regional recurrence in left psoas muscle and bilateral lung metastases as concluded by CT scan of chest, abdomen and pelvis. ${ }^{18} \mathrm{FDG}$ PET/CT was updated in January 2015 before starting chemotherapy and confirmed hypermetabolic local relapse and hypermetabolic lung lesions.

Due to impaired renal function with GFR $<60 \mathrm{ml} / \mathrm{min}$ (GFR $47 \mathrm{ml} / \mathrm{min}$ evaluated with MDRD formula), gemcitabine-carboplatin based first-line chemotherapy was started and the patient received 6 cycles of gemcitabine $1,000 \mathrm{mg} / \mathrm{m}^{2}$ on day 1 and 8 and carboplatin on day 1 (target area under curve of 4) every 21 days, ended in May 2015. After completion of 6 cycles, CT scan displayed an objective response according to RECIST 1.1 criteria (-53\% for target lesions) and ${ }^{18} \mathrm{FDG}$ PET/CT found a partial metabolic response. There were no extra-hematological grade 3-4 adverse events.

In August 2015, the patient was admitted for low back pain and left cruralgia. CT scan of Chest-Abdomen and pelvis 

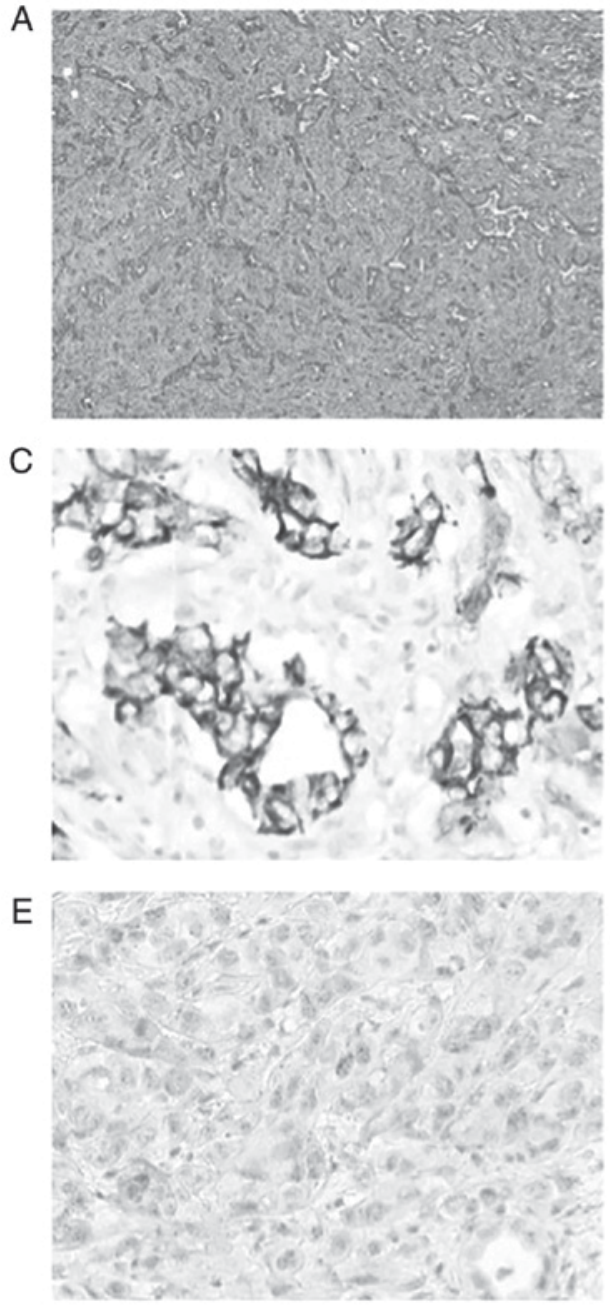

B

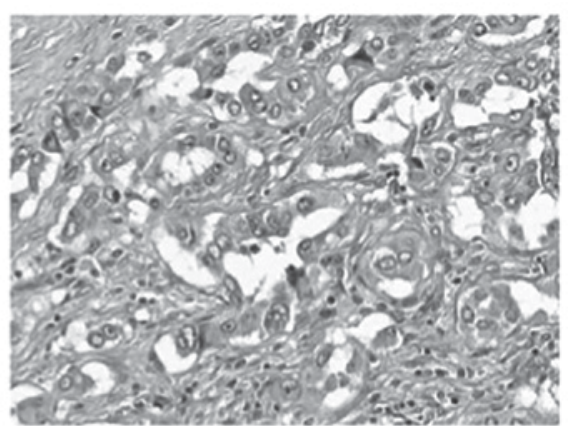

$\mathrm{D}$
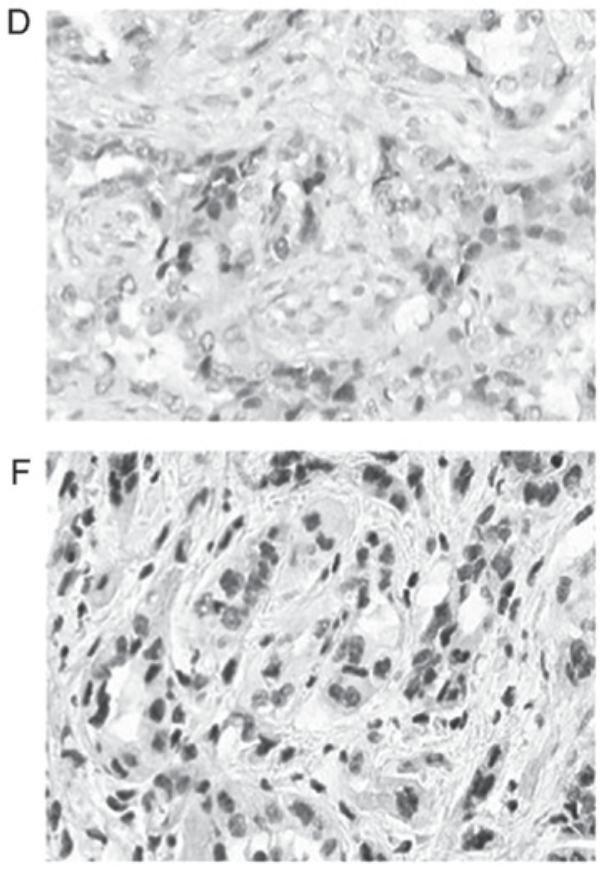

Figure 2. Histology section of collecting duct carcinoma and results of ancillary immunohistochemistry. Microscopic pictures showing a collecting duct carcinoma: Hematoxylin eosin saffron stain (A) low magnification x50 and (B) high magnification x300 and immunochemistry showing (C) an intense positivity of Cytokeratin 19 (x400), (D) a nuclear positivity of PAX8 (x400), (E) a negativity of P63 (x400), and (F) a conserved 1N1-1 (x400).

displayed rapid tumor progression compared to May 2015 with new lung metastases and progression of the other known lesions, including progression of the lesion in left psoas muscle. ${ }^{18} \mathrm{FDG}$ PET/CT was performed in order to have baseline functional imaging.

Despite a free interval between the end of first-line chemotherapy and disease progression less than 3 months, the same GC based-chemotherapy was resumed in September 2015. Patient received 6 new cycles until February 2016.

CT-scan evaluation between cycle 3 and 4 concluded an objective response according to RECIST 1.1 criteria (-43\% for target lesions). At the end-of-treatment CT scan, disease was stable compared to nadir $\mathrm{CT}$ scan with a remaining residual disease. ${ }^{18} \mathrm{FDG}$ PET/CT displayed residual hypermetabolic lesions. It was decided to stop chemotherapy and to follow-up the patient with CT scan of chest, abdomen and pelvis every 3 months.

During follow-up, the patient did not display any sign of progressive disease until June 2016 and target lesions remained stable. From September 2016 to May 2017, the patient developed again a late objective and confirmed response at each CT-scan evaluation, until complete remission in May 2017. Complete remission was confirmed with ${ }^{18}$ FDG-PET/CT in July 2017. No late side effects of chemotherapy were recorded.
From September 2016 to May 2017, no new treatment was added and no cytotoxic treatment was resumed. In April 2018, the patient was still free of disease with a total follow-up of near 4 years after diagnosis. He died in August 2018 in another medical center from acute renal and cardiac failures with no evidence of cancer relapse. The history of our patient is summarized in Fig. 3.

\section{Discussion}

To our knowledge, this is the first case to report a durable complete remission in metastatic renal CDC after GC-based chemotherapy. Moreover, we observed an unusual prolonged survival in this poor-prognosis disease.

Interestingly in our case, objective response was delayed with a complete remission occurring more than one year after the completion of the last chemotherapy cycle, while no other treatment was prescribed. This profile of delayed durable complete response was never described before in metastatic renal CDC and might imply role of immune system despite there is no evidence to support this interpretation. Recent data suggested the potential efficacy of immune checkpoint inhibitors targeting PD-1/PD-L1 pathway in metastatic renal 


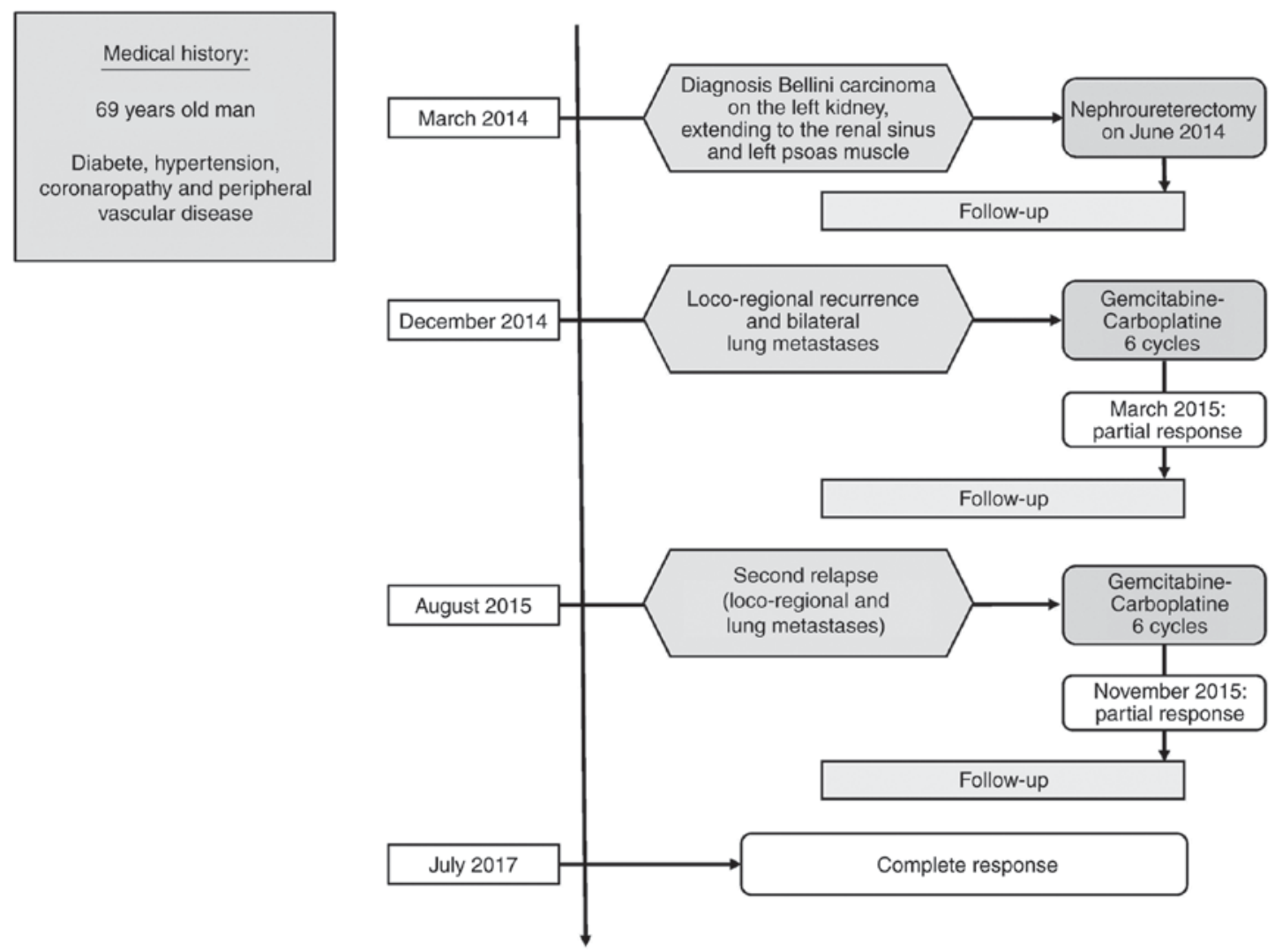

Figure 3. Medical history of the patient.

CDC $(14,15)$, and so the potential implication of immune response in the control of metastatic renal CDC.

Recent data have provided huge genomic profiling information on CDC, leading to a better understanding of the disease, and the identification of potential actionable targets. Malouf et al discovered that CDC displays a unique transcriptomic signature among kidney cancer subtypes (16). This pathognomonic transcriptomic signature is characterized by immunogenic and metabolic aberrations, indicating that targeting these processes might provide therapeutic options for patients. A series of 17 patients with CDC was studied by Pal et al Recurrent clinical relevant genomic alterations were detected and suggested a possible benefit from targeted therapy, such as mTOR inhibitors in patients with NF2 alterations (17).

In our case, after the completion of first-line chemotherapy, the free interval before disease new progression was less than 3 months and we paradoxically decided to resume the same GC-based chemotherapy. In other metastatic malignant tumors such as metastatic urothelial carcinoma, starting a new second line treatment would have been the best option. In our case, because of an objective response after first-line chemotherapy, a very good clinical and biological safety profile and because there was no evidence to support another chemotherapy regimen, we chose to resume the same GC-based chemotherapy.

Moreover, in our case, due to impaired renal function, carboplatin was chosen instead of cisplatin, usually more efficient than carboplatin, especially in metastatic urothelial carcinoma.

In the literature, we did not find case reporting durable complete remission of metastatic renal CDC.
As reported by the GETUG phase 2 trial, gemcitabine/platinbased chemotherapy is the only regimen that had prospectively shown efficacy in metastatic CDC, supporting this regimen as a standard-of-care in metastatic CDC despite only 23 patients were evaluated in this prospective trial (6). Gemcitabine/platin-based chemotherapy was able to provide a durable and complete response in our patient.

In rare tumors such as metastatic CDC, prospective data are usually limited and disease management is also supported by low level of evidence data, including case reports and retrospective series. We believe that our results can contribute with other published data to confirm GC-based chemotherapy as a standard-of-care in first line treatment of metastatic renal CDC.

Actually, one clinical trial is in progress, assessing antiangiogenic treatment with bevacizumab combined to gemcitabine/platin-based chemotherapy (BEVABEL GETUG-AFU phase 2 Trial (NCT02363751)). Results are not yet available.

In conclusion, we report a durable and delayed complete remission in metastatic renal CDC more than 1 year after completion of GC-based chemotherapy. Regarding to the literature and our experience, this supports platin salt and gemcitabine combination as the best regimen in first-line treatment of metastatic renal CDC. Rechallenging the same chemotherapy after rapid progression could be an option.

\section{Acknowledgements}

Not applicable. 


\section{Funding}

No funding was received.

\section{Availability of data and materials}

The datasets used and/or analyzed during the current study are available from the corresponding author on reasonable request.

\section{Authors' contributions}

DD and JLD analyzed and interpreted the patient data regarding the oncology disease and its progression. CD performed the histological examination of the kidney tumor and was a major contributor in writing the manuscript. RB and VD were the surgeons of the patient and critically revised the manuscript. $\mathrm{PH}$ acquired the computed tomography images. SS and FD made substantial contributions to the interpretation of data, were involved in writing the discussion and critically revised the manuscript. All authors read and approved the final manuscript.

\section{Ethics approval and consent to participate}

Not applicable.

\section{Patient consent for publication}

The patient gave oral permission for the publication of these data. All reasonable attempts were made to contact the next of kin for written permission, but this proved not to be possible.

\section{Competing interests}

JLD has received speaker fees from Janssen-Cilag, BMS, Astellas, Pfizer and has received fees as an advisory board member for Janssen-Cilag, BMS and Sanofi.

\section{References}

1. McDougal W, Wein A, Kavoussi L, Novick A, Partin A, Peters C and Ramchandani P: Malignant renal tumors. In: Campbell-walsh Urology 10th Edition Review. Elsevier Health Sciences, pp1436, 2011.

2. Andola SK, Laheru V and Patil S: Collecting duct carcinoma of the kidney. J Sci Res 6: 46-48, 2013.

3. Méjean A, Rouprêt M, Larousserie F, Hopirtean V, Thiounn N and Dufour B: Is there a place for radical nephrectomy in the presence of metastatic collecting duct (Bellini) carcinoma? J Urol 169: 1287-1290, 2003.
4. Peyromaure M, Thiounn N, Scotté F, Vieillefond A, Debré B and Oudard S: Collecting duct carcinoma of the kidney: A clinicopathological study of 9 cases. J Urol 170: 1138-1140, 2003.

5. Tokuda N, Naito S, Matsuzaki O, Nagashima Y, Ozono S and Igarashi T; Japanese Society of Renal Cancer: Collecting duct (Bellini duct) renal cell carcinoma: A nationwide survey in Japan. J Urol 176: 40-43, 2006.

6. Oudard S, Banu E, Vieillefond A, Fournier L, Priou F, Medioni J, Banu A, Duclos B, Rolland F, Escudier B, et al: Prospective multicenter phase II study of gemcitabine plus platinum salt for metastatic collecting duct carcinoma: Results of a GETUG (Groupe d'Etudes des Tumeurs Uro-Génitales) study. J Urol 177: 1698-1702, 2007.

7. Dason S, Allard C, Sheridan-Jonah A, Gill J, Jamshaid H, Aziz T, Kajal B and Kapoor A: Management of renal collecting duct carcinoma: A systematic review and the McMaster experience. Curr Oncol 20: e223-e232, 2013

8. Barrascout E, Beuselinck B, Ayllon J, Bättig B, Moch H, Teghom $\mathrm{C}$ and Oudard S: Complete remission of pulmonary metastases of Bellini duct carcinoma with cisplatin, gemcitabine and bevacizumab. Am J Case Rep 13: 1-2, 2012.

9. Pécuchet N, Bigot F, Gachet J, Massard C, Albiges L, Teghom C, Allory Y, Méjean A, Escudier B and Oudard S: Triple combination of bevacizumab, gemcitabine and platinum salt in metastatic collecting duct carcinoma. Ann Oncol 24: 2963-2967, 2013

10. Mennitto A, Verzoni E, Peverelli G, Alessi A and Procopio G: Management of metastatic collecting duct carcinoma: An encouraging result in a patient treated with cabozantinib. Clin Genitourin Cancer 16: e521-e523, 2018.

11. Selli C, Amorosi A, Vona G, Sestini R, Tmvaglini F, Bartoletti R and Orlando C: Retrospective evaluation of c-erbb-2 oncogene amplification using competitive PCR in collecting duct carcinoma of the kidney. J Urol 158: 245-247, 1997.

12. Bronchud MH, Castillo S, Escriva de Romaní S, Mourelo S, Fernández A, Baena C, Murillo J, Julia JC, Esquius J, Romero R and Andreu X: HER2 blockade in metastatic collecting duct carcinoma (CDC) of the kidney: A case report. Onkologie 35: 776-779, 2012.

13. Procopio G, Verzoni E, Iacovelli R, Colecchia M, Torelli T and Mariani L: Is there a role for targeted therapies in the collecting ducts of Bellini carcinoma? Efficacy data from a retrospective analysis of 7 cases. Clin Exp Nephrol 16: 464-467, 2012.

14. Koshkin VS, Barata PC, Zhang T, George DJ, Atkins MB, Kelly WJ, Vogelzang NJ, Pal SK, Hsu J, Appleman LJ, et al: Clinical activity of nivolumab in patients with non-clear cell renal cell carcinoma. J Immunother Cancer 6: 9, 2018.

15. Mizutani K, Horie K, Nagai S, Tsuchiya T, Saigo C, Kobayashi K, Miyazaki T and Deguchi T: Response to nivolumab in metastatic collecting duct carcinoma expressing PD-L1: A case report. Mol Clin Oncol 7: 988-990, 2017.

16. Malouf GG, Compérat E, Yao H, Mouawad R, Lindner V, Rioux-Leclercq N, Verkarre V, Leroy X, Dainese L, Classe M, et al: Unique transcriptomic profile of collecting duct carcinomas relative to upper tract urothelial carcinomas and other kidney carcinomas. Sci Rep 6: 30988, 2016.

17. Pal SK, Choueiri TK, Wang K, Khaira D, Karam JA, Van Allen E, Palma NA, Stein MN, Johnson A, Squillace R, et al: Characterization of clinical cases of collecting duct carcinoma of the kidney assessed by comprehensive genomic profiling. Eur Urol 70: 516-521, 2016. 